\title{
BIM NO PROCESSO DE PROJECTO ARQUITECTÓNICO: UMA PROPOSTA DE POTENCIALIZAÇÃO
}

\author{
Josyanne Giesta $^{(1)}$, Karine Morais ${ }^{(1)}$, Maytê Cunha ${ }^{(2)}$, Rayanne Câmara ${ }^{(2)}$ \\ (1) Instituto Federal de Educação, Ciência e Tecnologia do Rio Grande do Norte, Natal \\ (2) Universidade Federal do Rio Grande do Norte, Natal
}

\begin{abstract}
Resumo
O processo de projeto arquitetónico (PPA) é uma das etapas mais complexas durante a construção de uma edificação, tendo em vista a necessidade de se encontrar soluções para problemas que possam existir futuramente e que sejam compatíveis com as demais disciplinas envolvidas e com as necessidades do cliente. Neste âmbito, o Building Information Modeling (BIM), ao ser inserido nesse processo, proporciona uma diminuição de erros, uma previsão das soluções escolhidas e a integração e troca de informações entre todos os profissionais envolvidos na construção, juntamente com o contratante. Desta maneira, tendo em vista a necessária atualização no PPA vigente, foram realizados estudos de caso em quatro escritórios de arquitetura em Natal, Rio Grande do Norte, os quais permitiram a verificação do processo de projetação utilizados nestes escritórios, avaliando-os qualitativamente. A partir dessa avaliação, desenvolveu-se um modelo beta para o PPA com a inserção do BIM que foi aperfeiçoado posteriormente, tendo como base uma validação feita pelos mesmos profissionais. Portanto, tendo em vista a implementação do BIM dentro desses escritórios e a adaptação dos mesmos, o presente trabalho busca apresentar uma proposta de processo de projeto arquitetónico com o BIM, objetivando a potencialização das funcionalidades deste.
\end{abstract}

\section{Introdução}

A Modelagem da Informação da Construção ou Building Information Modeling (BIM) é uma nova abordagem metodológica para processos de desenvolvimento do ambiente contruído, abrangendo projeto, construção, gerenciamento e manutenção de edificações e infraestrutura. Quando implementada de forma plena, todos os agentes envolvidos podem aceder, ao mesmo tempo, informações sobre o escopo de projeto, cronogramas e orçamentos que são de alta qualidade, confiáveis, integrados e totalmente coordenados [1].

Desta maneira, o BIM implica mudanças no processo de projeto, construção e acompanhamento do ciclo de vida do edifício, com novos processos de projeto, baseados na coordenação, na interoperabilidade, no compartilhamento e no reuso de informações. No campo de projeto, implica redistribuir os esforços da atividade dos projetistas, mudando a estrutura da ação 
projetual [2]. Um dos ganhos percetíveis é que o modelo de construção produzido por uma ferramenta BIM pode dar suporte a múltiplas vistas diferentes dos dados contidos dentro de um conjunto de desenhos, incluindo 2D e 3D [3]. Dessa forma, as vistas não são mais desenhadas e sim extraídas como as demais informações do modelo, otimizando, assim o processo de projeto arquitetónico (PPA).

No entanto, a implementação do BIM deve ser bem planejada e devidamente documentada a fim de se obter melhores resultados. A adoção descoordenada, sem liderança ou regulamentação leva a expectativas variadas, diminuindo, assim, o cumprimento dos benefícios potenciais para todas as organizações no processo de construção [4]. Nesse sentido, para que um escritório de arquitetura inicie seu processo de implementação do BIM é importante que o mesmo tenha conhecimento dos seus fluxos de trabalho, seus colaboradores e respetivas funções e sobretudo que identifique o que mudará no seu PPA com a inserção do BIM.

[5] através de um levantamento da produção científica brasileira sobre BIM, no intervalo de 2000 a 2015, revelaram que "processo de projeto" se encontra como a quarta palavra-chave mais utilizada entre onze selecionadas em teses e dissertações, indicando que o tema é de grande interesse e relevância para a comunidade acadêmica. Apesar disso, entende-se que mais pesquisas precisam ser realizadas, sobretudo que espelhem um contexto local, visto a grande desigualdade tecnológica e científica ainda existente no país.

Nesse cenário, o presente trabalho tem como objetivo desenvolver um processo de projeto arquitetónico com base nas análises realizadas pelos profissionais dos escritórios de arquitetura entrevistados. PPA este que seja o mais compatível possível com o perfil dos escritórios de arquitetura natalenses, de modo a favorecer o processo de implementação do BIM local.

\section{BIM no processo de projeto arquitetónico}

O projeto arquitetónico pode ser definido como a concretização de uma ideia, um espaço imaginado. Também é o processo pelo qual uma obra de arquitetura é concebida, ou seja, é uma representação de como será construído determinado ambiente.

O objetivo do processo de projeto é transformar um determinado problema em solução a partir de um conjunto de procedimentos e etapas de diferentes complexidades. Inicia-se pela exploração e pelo reconhecimento do problema, procurando a definição da alternativa mais adequada como solução, dando ênfase ao racional, ao funcional e ao criativo, bem como obedecendo aos critérios exigidos por normas e legislações pertinentes.

O processo de projeto é, essencialmente, uma sequência de aprimoramentos em um conjunto de informações a ser transmitido para as fases subsequentes [6]. A Figura 1 apresenta um exemplo de processo de projeto tradicional.

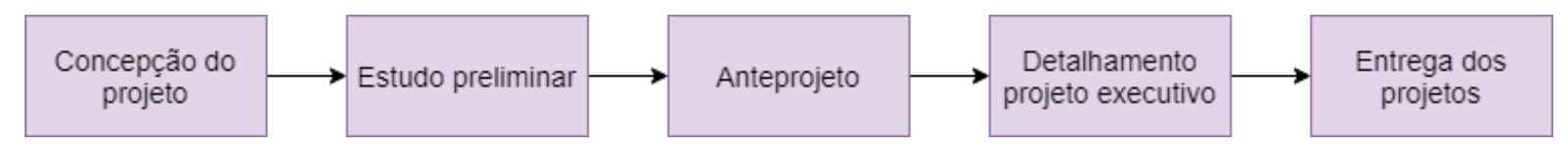

Figura 1: Processo de projeto tradicional (Adaptado de [7]).

A mudança no processo de projetar baseado em BIM é significativa e se reflete diretamente nos escritórios de arquitetura, uma vez que estes iniciam o processo e, em muitos casos, mediam e coordenam as demais especialidades [7]. 
Pesquisa realizada em escritórios de arquitetura do Rio de Janeiro, São Paulo e Curitiba apontou que o uso do BIM se concentra nas etapas de projeto: Estudos preliminares (53\%), Anteprojeto (50\%), Projeto Legal (56\%) e Projeto Executivo (47\%) [8].

\section{Método}

O método aplicado na pesquisa foi o Estudo de caso, que se caracteriza como uma pesquisa empírica que busca melhor compreender um fenômeno contemporâneo, normalmente complexo, no seu contexto real [9]. Ainda de acordo com os autores, esse método é adequado para investigar problemas complexos dentro do contexto em que ocorrem.

Para tanto, foram definidas as cinco etapas propostas por [10]: a) delineamento da pesquisa; $b$ ) desenho da pesquisa; c) preparação e coleta dos dados; d) análise dos casos e entre os casos; e e) elaboração dos relatórios (Figura 2). Cabe destacar que as etapas d) e e) não podem ser isoladas, elas ocorrem de forma paralela.

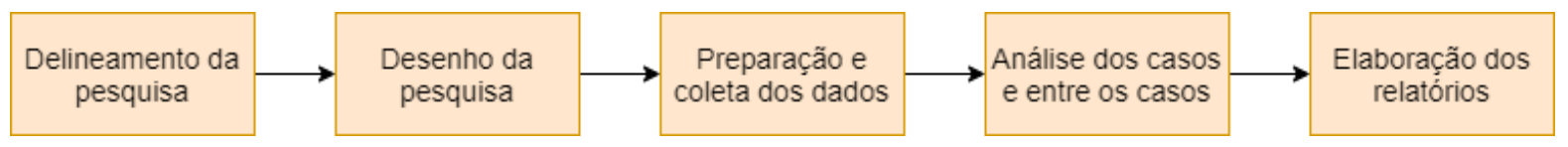

Figura 2: Etapas do Estudo de caso.

Tendo a etapa delineamento da pesquisa como produto final a revisão bibliográfica, após a definição dos objetivos e as questões de pesquisa, iniciou-se o levantamento bibliográfico, que consistiu em uma pesquisa bibliográfica sem sistematização rigorosa nas buscas, o que permitiu destacar ideias de outros autores para fundamentar o presente trabalho, de forma a construir uma estrutura base para a pesquisa.

$\mathrm{Na}$ etapa de desenho da pesquisa, foram definidos os quatro Estudos de Caso e desenvolvido o protocolo, que orientou as atividades de coleta dos dados.

A etapa de preparação e coleta dos dados, teve o contato com os casos selecionados, e foram marcadas as entrevistas a serem realizadas em dois momentos distintos: a) preliminar para caracterização do escritório e obtenção de informações acerca do processo de projeto desenvolvido; b) definitivo para a validação do modelo de processo de projeto BIM proposto. Com relação aos locais de aplicação das entrevistas, nos dois momentos foram nos próprios escritórios de arquitetura.

Com a etapa de coleta de dados realizada, iniciou-se a análise dos casos e elaboração dos relatórios, que resultou em uma proposta modelo alfa, que corresponde ao modelo beta refinado.

\section{Estudos de Caso}

Tendo como objetivo a compreensão do processo de projeto arquitetónico, realizaram-se entrevistas com quatro escritórios de arquitetura de Natal, Rio Grande do Norte - Alicerce Arquitetura, Espaço Quatre, Matiz Escritório de Arquitetura e Primo Piano Arquitetos. De modo geral, a Figura 3 apresenta o processo de projeto arquitetónico usual. 


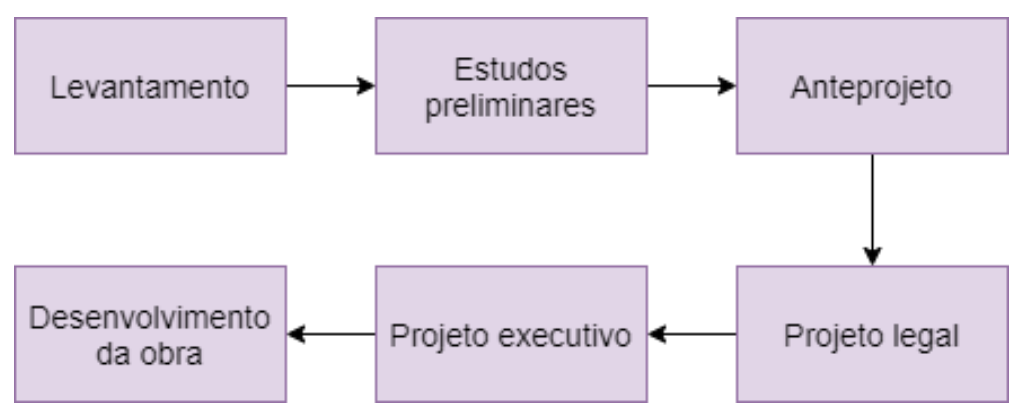

Figura 3: Etapas do processo de projeto arquitetónico.

A partir da metodologia usada nos escritórios, foram realizados estudos sobre o BIM, de modo a identificar como o mesmo poderia ser implementado nestas empresas. Por meio disso, foi desenvolvida uma proposta de processo de projeto com BIM, a qual foi analisada e validada pelos mesmos profissionais, em um segundo momento. Tais validações, possibilitaram a extração dos principais conceitos e aspetos que o processo de projetação deve conter na visão destes e como a solução apresentada poderia ser reformulada para uma melhor adaptação ao mercado de arquitetura natalense.

\subsection{Proposta modelo beta}

Após o primeiro momento de entrevistas com os profissionais dos escritórios, foi desenvolvido um processo metodológico, baseando-se em dados previamente coletados, tendo como objetivo a utilização do BIM de forma integral, e trazendo o total aperfeiçoamento de suas funcionalidades, permitindo, também, um total funcionalismo prático do processo, como ilustra a Figura 4.

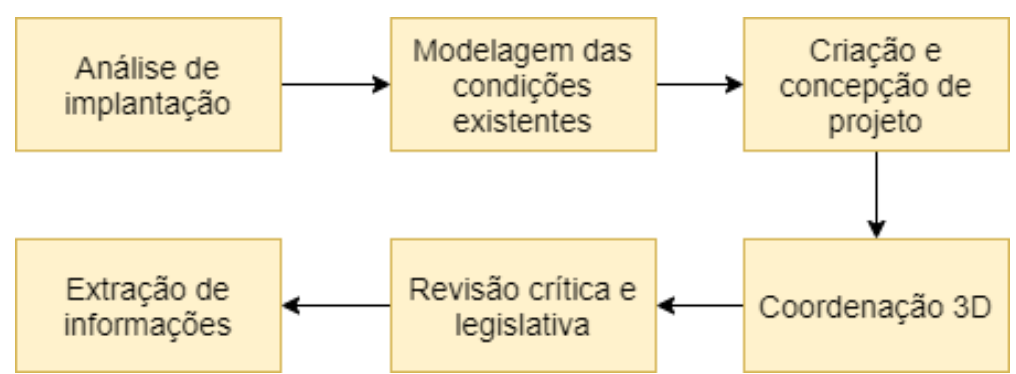

Figura 4: Etapas do processo de projeto arquitetónico com o BIM (modelo beta).

Visando a otimização de tal metodologia, a mesma foi submetida a validação, realizada pelos profissionais anteriormente entrevistados, em um segundo momento de entrevistas, com o objetivo de identificar a aplicabilidade da mesma nos escritórios de arquitetura de Natal, e, também, para que os problemas existentes nela fossem evidenciados, permitindo a correção destes.

\subsection{Validação do modelo beta}

\subsubsection{Alicerce Arquitetura}

Durante a apresentação da proposta para a arquiteta e gestora do escritório, o primeiro aspeto apontado acerca da metodologia foi a boa organização da mesma e a fácil compreensão de seu 
desenvolvimento, sendo apresentada como dificultosa apenas a etapa de conceção de projeto, pois, segundo a entrevistada, as ferramentas BIM coíbem o processo criativo do projetista. Quanto aos principais requisitos necessários para o fundamento do BIM, foram exibidos comentários mais incisivos.

Mesmo sendo consideradas mudanças pertinentes, uma das principais dificuldades expostas foram as complicações que poderiam surgir com a divisão de funções, tendo em vista a presença de apenas dois profissionais atuantes no escritório: o arquiteto coordenador e o estagiário, situação recorrente nos escritórios de arquitetura de Natal. Desta maneira, o estagiário executaria as atividades de modelagem e o arquiteto coordenador conceberia e revisaria os aspetos modelados.

Além disso, foi apresentado também como um impasse o complexo desenvolvimento do treinamento de profissionais do escritório, tendo em vista a difícil adaptação dos mesmos a nova filosofia de trabalho. Tal atividade complica a implementação do BIM, pois se necessita de um maior período de tempo para aprender sobre a metodologia e todos os conceitos que rodeiam esta, adequando-se para os fluxos de trabalho da empresa.

\subsubsection{Espaço Quatre}

Objetivando a validação da solução criada, foi realizada a apresentação da metodologia e de todos os requisitos que a circundam para a arquiteta e também diretora de projetos do escritório. A arquiteta, por ter um conhecimento mais abrangente acerca da filosofia BIM e uma experiência consolidada com a mesma, foi mais incisiva nas críticas à metodologia. Foram apresentadas novas etapas no processo de projetação proposto e recomendadas novas denominações às etapas apresentadas, como mostra a Figura 5 (a).

Quanto aos requisitos necessários para o bom funcionamento do escritório com o uso do BIM, foi sugerida a adição de padronização dos processos, ou seja, a definição de parâmetros que moldariam o perfil da empresa, voltada tanto para os fatores externos quanto para os fatores internos, enfatizando-se a importância da exploração desse conceito e de sua abrangência dentro da empresa. Pontos como a existência de um bom parque tecnológico e a capacitação de profissionais foram apontados como essenciais, mas que também deveriam se enquadrar nos padrões estabelecidos.

Por fim, foi identificada a necessidade de reorganização dos recursos humanos da empresa, visando um melhor fluxo de trabalho. Desta maneira, foi recomendada a criação de uma hierarquia na qual o arquiteto coordenador atuaria como coordenador geral do modelo, concebendo e revisando, e os demais colaboradores realizariam as atividades de modelagem, complementação de desenho e desenvolvimento de bibliotecas digitais; tais funções também poderiam ser executadas por um único profissional.

\subsubsection{Matiz Escritório de Arquitetura}

$\mathrm{Na}$ apresentação da proposta para a arquiteta e gerente do escritório os procedimentos exibidos no processo de projetação foram muito elogiados, principalmente por sua organização e fácil entendimento. Pontuando os fatores que mais dificultam a implementação do BIM, a entrevistada apresentou em primeiro lugar, por ser um fator essencial para a existência da metodologia, a infraestrutura computacional, tendo em vista o elevado custo da mesma.

Além disso, enquadrando-se como segundo fator apresentado, foi exposta a dificuldade de se compreender a filosofia BIM e suas funcionalidades em sua totalidade, ocasionando uma deficiência no aperfeiçoamento da metodologia e do fluxo de trabalho existente. Havendo 
também um problema com os prazos estabelecidos para o cliente, pois, com o déficit no discernimento necessário sobre o novo processo e na organização de seus procedimentos, o período de tempo se torna maior do que o que já estava consolidado com as práticas anteriores. Por fim, a estrutura dos recursos humanos da empresa foi enfatizada, exibindo-se a inviabilidade de aplicação devido ao desequilíbrio das funções apresentadas. Tendo em vista a existência de apenas dois colaboradores no escritório, sendo estes: o arquiteto coordenador, o qual se encarregaria de conceber e revisar o projeto, e o estagiário, que desenvolveria a modelagem, seu detalhamento e ainda seria o responsável pela organização da biblioteca digital; verificando-se, deste modo, a sobrecarga de atividades sobre este.

\subsubsection{Primo Piano Arquitetos}

Visando a validação da proposta de processo de projeto criada, foi realizada a apresentação da mesma e de todos os requisitos que a circundam ao arquiteto e coordenador de projetos do escritório.

Tendo em vista a dificuldade de transição entre as metodologias e de identificação, num primeiro momento, das etapas apresentadas, foi apontada a necessidade de comparação e da instituição da relação entre a metodologia com o BIM e a NBR 13531-95.

Foi recomendada também uma mudança no diagrama de apresentação dos procedimentos, transferindo a etapa de Coordenação 3D para o centro do processo, caracterizando-a como essencial para o desenvolvimento do projeto por estar presente em todas as fases.

Para uma melhor estruturação do escritório, foram exibidos os aspectos essenciais para o bom funcionamento da metodologia BIM por nível de importância, sendo mencionada, principalmente, a necessidade de um bom parque tecnológico além da capacitação de profissionais, quesito bastante salientado pelo entrevistado. E, permitindo a atualização constante dos profissionais, foi recomendada a criação de um manual de boas práticas próprio do escritório, no qual seriam especificados os fluxos seguidos pela empresa e os principais conceitos adotados pela mesma.

Por fim, a quantidade de colaboradores também foi, novamente, apresentada como um impasse para implantação da metodologia. Desta forma, foi recomendada a aplicação de especialidades BIM dentro do escritório, tais como: Modelador BIM, Facilitador BIM, Desenvolvedor de Bibliotecas Digitais e Gerente BIM. Tal esquematização foi representada pela Figura 5 (b).

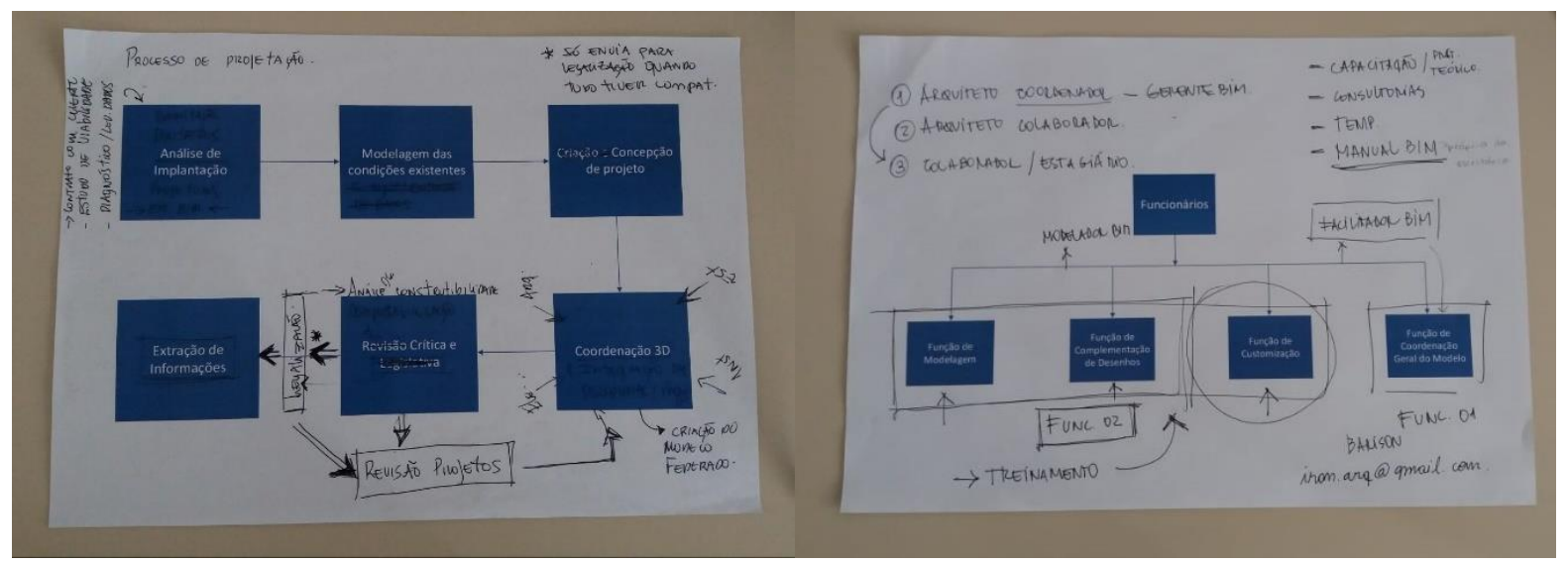

Figura 5: (a) Novas etapas (b) Perfil dos colaboradores. 


\section{Resultados e discussão}

Tendo em vista a validação feita pelos profissionais dos quatro escritórios de arquitetura, os quais expuseram suas opiniões sobre o processo metodológico apresentado (modelo beta), fazendo recomendações acerca do mesmo, foi realizada uma análise, a qual permitiu o refinamento do modelo. Desta maneira, são apresentadas as modificações feitas nas etapas já existentes e inseridos novos conceitos a estas. Bem como são identificados quatro requisitos para implementação do BIM.

\subsection{Inserção e reorganização de etapas - modelo alfa}

Considerando-se a complexidade apresentada pelo desenvolvimento do processo de projetação, foram necessárias alterações na organização da metodologia e a inserção de novos procedimentos que completassem as lacunas apresentadas no modelo beta. Desta maneira, são apresentadas as etapas e modificações realizadas que resultaram no modelo alfa (Figura 6).

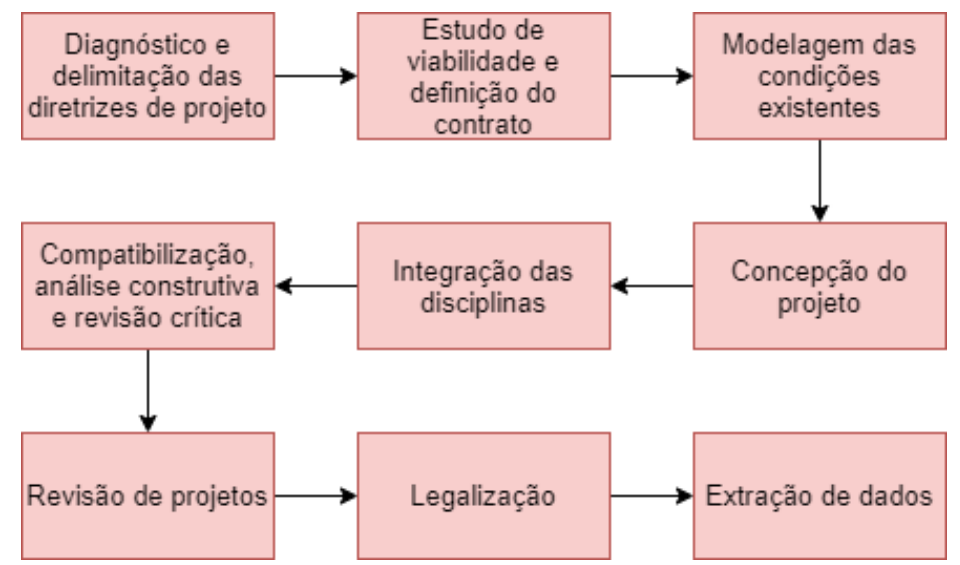

Figura 6: Etapas do processo de projeto arquitetónico com BIM (modelo alfa) [11].

\subsubsection{Diagnóstico e delimitação das diretrizes de projeto}

Essa primeira etapa do fluxo, substitui a "análise de implantação", acrescentando mais rigor nas definições referentes as diretrizes de projeto.

\subsubsection{Estudo de viabilidade e definição do contrato}

Essa etapa é nova, e tem como principal objetivo a formatação do contrato, o qual deve definir de forma clara os entregáveis e características como LOD e LOI do modelo.

\subsubsection{Modelagem das condições existentes - Conceção do projeto}

Essas duas etapas não sofreram alterações.

\subsubsection{Integração das disciplinas - Compatibilização, análise construtiva e revisão crítica - Revisão de projetos - Legalização}

As etapas "Coordenação 3D" e "Revisão crítica e legislativa" do modelo beta foram substituídas por quatro etapas, em razão de existir entre as atividades uma relação de precedência. 
Na etapa de Integração das disciplinas será realizada a integração entre o projeto arquitetônico e os projetos de engenharia, lembrando que para tanto será relevante que os projetos tenham interoperabilidade entre eles, ou que se encontrem em formato aberto (IFC).

A etapa de Compatibilização, análise construtiva e revisão crítica tem como objetivo a identificação de interferências entre os projetos, a verificação da possibilidade de execução dos detalhes construtivos propostos, bem como a aprovação do projeto por parte do cliente. Essa etapa do fluxo BIM é muito importante e apresenta um diferencial com relação ao fluxo tradicional, pois permite que de forma antecipada se identifique problemas e defina soluções que só seriam percebidas durante a execução da obra.

A etapa de Revisão de projetos corresponde a cada responsável por sua disciplina fazer as correções necessárias, visto que apesar do BIM se tratar de um processo colaborativo, ainda existe toda a questão da autoria do projeto, que continua tendo que ser respeitada.

Com relação a etapa Legalização, esta etapa só deve ocorrer quando os projetos estiverem totalmente compatibilizados e revisados, visto que passarão pelo processo de aprovação por parte dos órgãos responsáveis, lembrando que, nesse quesito, ainda existe um certo descompasso quando se trata do BIM e os órgãos públicos no país, pois muitas regiões ainda operam no sistema tradicional, o que acaba por implicar em entregar ao órgão para análise a documentação ainda em CAD.

\subsubsection{Extração de dados}

Essa etapa final mudou de nome, anteriormente era "Extração de informações", para simbolizar uma amplitude maior, incluindo dados geométricos e não geométricos.

\subsection{Requisitos para implementação do BIM}

Considerando-se a estrutura dos escritórios de arquitetura natalenses, foram definidos quatro requisitos relevantes para a implementação do BIM (Figura 7), a saber: definição de parque tecnológico com aquisição de hardwares, definição de perfis e papéis dos colaboradores, preocupação com capacitação dos profissionais respeitando os perfis pré-definidos, e sobretudo a padronização de processos.

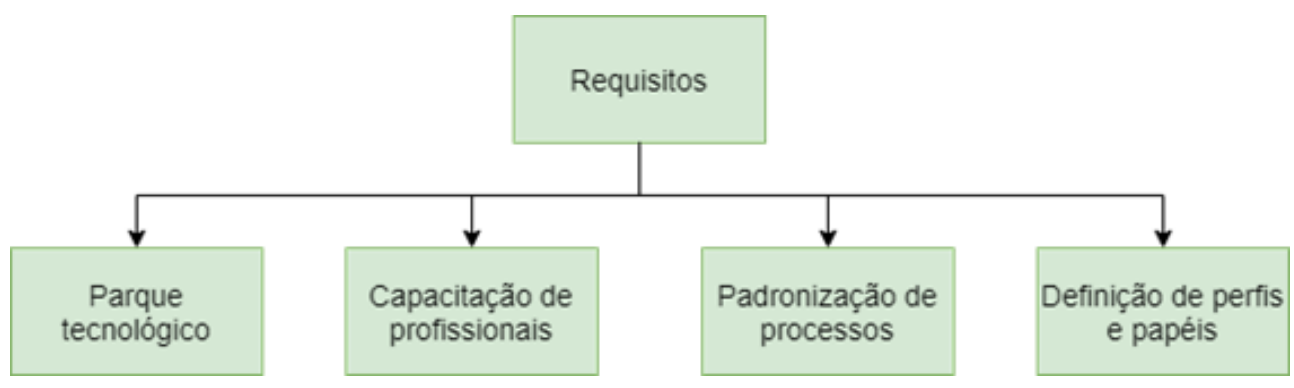

Figura 7: Requisitos para a implementação do BIM.

\subsubsection{Parque tecnológico e Capacitação de profissionais}

Com relação ao parque tecnológico e a capacitação dos profissionais, geralmente para essas definições é importante a ajuda de uma consultoria específica, de modo que as escolhas sejam as mais adequadas possíveis. 


\subsubsection{Padronização de processos}

Identificando-se como um dos principais requisitos para a implementação do BIM, a padronização dos processos se caracteriza pela definição de parâmetros que devem moldar o perfil da empresa. Os fatores que compõem esta diretriz, são: os fatores externos, referentes ao que será entregue pelo escritório, e os fatores internos, referentes aos perfis dos profissionais e aos fluxos estabelecidos na empresa.

\subsubsection{Definição de perfis e papéis}

Ainda com base nas entrevistas, foram definidos quatro perfis de profissionais BIM, lembrando que, em situações onde o escritório de arquitetura possua um número menor de profissionais, as atribuições podem e devem ser agrupadas, tentando ao máximo não sobrecarregar um único colaborador.

O gerente BIM é responsável pelo processo BIM, cabendo ao mesmo a definição da equipe, seus perfis e papéis. Com relação ao modelador BIM, ele será o responsável pela tomada de decisões no projeto, como definições de níveis, de eixos, entre outras. O facilitador BIM fará as finalizações e o detalhamento do modelo, enquanto que o desenvolvedor BIM é aquele responsável pelo desenvolvimento da biblioteca de componentes BIM (Figura 8).

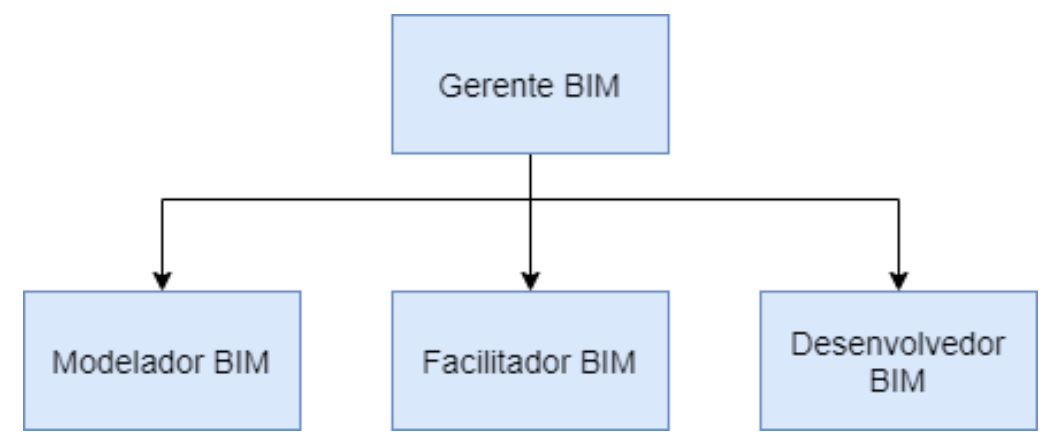

Figura 8: Definição de perfis BIM (Adaptado de [12]).

\section{Considerações finais}

Objetivando apresentar uma proposta de processo de projeto arquitetônico (PPA) com BIM foram realizados quatro estudos de caso, que permitiram desenvolver e refinar o modelo proposto. $\mathrm{O}$ modelo proposto contempla as principais etapas a serem desenvolvidas de modo a proporcionar um melhor fluxo no processo de projeto arquitetônico, tornando-o mais dinâmico entre os profissionais envolvidos, satisfazendo tanto estes quanto os contratantes, possibilitando ainda, a diminuição de erros na projetação, a otimização do tempo e a diminuição de gastos na obra.

Como limitações pode-se destacar a localização dos estudos de caso, visto que todos são escritórios da capital - Natal e o fato de que o fluxo desenvolvido não foi testado na prática. Nesse contexto, tem-se como proposta para pesquisas futuras que o modelo seja testado em escritórios que ainda não iniciaram o processo de implementação do BIM, bem como que esse modelo seja estudado em escritórios de arquitetura localizados em outras cidades do Estado, objetivando se necessário fazer modificações no modelo, de forma a adequá-lo a outras realidades. 


\section{Referências}

[1] M. B. Barison; E. T. Santos, "O papel do arquiteto em empreendimentos desenvolvidos com a tecnologia BIM e as habilidades que devem ser ensinadas na universidade," Gestão e Tecnologia de Projetos, v.11, n.1, p. 103-120, Jan/Jun 2016. Doi: 10.11606/gtv.v11i102708.

[2] A. P. C. Pereira, "Modelagem da Informação de Construção na fase de projeto: proposta de plano de execução BIM para a SUMAI/UFBA," Tese de Doutoramento, Programa de Pós-Graduação em Arquitetura e Urbanismo, Universidade Federal da Bahia, Salvador, Brasil, 2017.

[3] C. M. Eastman; P. Teicholz; R. Sacks; K. Liston, Manual de BIM: um guia de modelagem da informação da construção para arquitetos, engenheiros, gerentes, construtores $e$ incorporadores. Porto Alegre: Bookman, 2014.

[4] U. Gurevich; R. Sacks, "Development of a BIM Adoption Impact Map," in Joint Conference on Computing in Construction (2017), Heraklion, Greece, 2017. Disponível em: https://www.researchgate.net/publication/318656920. Acesso em 18 de julho de 2020.

[5] F. A. Machado; R. C. Ruschel; S. Scheer, "Análise da produção científica brasileira sobre a Modelagem da Informação da Construção,” Ambiente Construído, v. 17, n. 4, p. 359-384, out./dez. 2017. ISSN 1678-8621 Associação Nacional de Tecnologia do Ambiente Construído. http://dx.doi.org/10.1590/s1678-86212017000400202

[6] C. Ayres Filho, "Acesso ao modelo integrado do edifício," Dissertação de Mestrado, Universidade Federal do Paraná, Programa de PósGraduação em Construção Civil do Setor de Tecnologia, Curitiba, Brasil, 2009.

[7] M. A. L. Garbini; D. Q. Brandão, "Proposta de modelo para implantação de processo de projeto utilizando o conceito BIM em escritórios de arquitetura," Gestão e Tecnologia de Projetos, v. 9, n. 1, p. 7-24, jan./jun. 2014. http://dx.doi.org/10.11606/gtp.v9i1.89990

[8] L. L. A. de Souza; S. R. L. Amorim; A. de M. Lyrio, "Impactos do uso do BIM em escritórios de arquitetura: oportunidades no mercado imobiliário," Gestão e Tecnologia de Projetos, v.4, n.2, p. 26-53, Nov 2009. ISSN 19811543.

[9] A. Dresch; D. P. Lacerda; J. A. V. Antunes Júnior, Design Science Research: método de pesquisa para avanço da ciência e tecnologia. Porto Alegre: Bookman, 2015.

[10] R. M. Branski; R. A. C. Franco; O. F. Lima Jr., "Metodologia de estudo de casos aplicada à logistica," in XXIV ANPET Congresso de Pesquisa e Ensino em Transporte (2010), Salvador, Brasil, 2010. Disponível em: https://www.researchgate.net/publication/277598822_METODOLOGIA_DE_ESTUDO_ DE_CASOS_APLICADA_A_LOGISTICA. Acesso em 21 de julho de 2020.

[11] J. P. Giesta; K. S. Morais; J. M. F. O. M. Pinto; T. C. Nascimento; A. Costa Neto, "Proposta de inserção do BIM no processo de projeto arquitetônico," in CIAC2019 - Conference on Automation Innovation in Construction (2019), Leiria, Portugal, 2019. Disponível em: https://sites.ipleiria.pt/ciac/proceedings/. Acesso em 20 de julho de 2020.

[12] K. da S. Morais; J. P. Giesta, "Proposta de inserção do BIM no processo de projeto arquitetônico," in IV Congresso Nacional de Construção de Edifícios - CONACED (2019), João Pessoa, Brasil, 2019. 